\title{
Surgical treatment of Dermatofibrosarcoma protuberans with a free anterolateral thigh (ALT) flap in an adolescent
}

\author{
Ernst L Möller, ${ }^{1}$ Francois Malherbe, ${ }^{2}$ Christopher E Price ${ }^{1}$ \\ ${ }^{1}$ Department of Plastic and Reconstructive Surgery, Groote Schuur Hospital, University of Cape Town, South Africa \\ ${ }^{2}$ Department of General Surgery, Groote Schuur Hospital, University of Cape Town, South Africa
}

Corresponding author: Ernst Möller (ernstmoller_10@hotmail.com)

Summary: A 16-year-old male presented with a 2-year history of a 5 x $5 \mathrm{~cm}$ dermatofibrosarcoma protuberans (DFSP) on the medial aspect of his right lower leg. Wide local excision (WLE) with a $2 \mathrm{~cm}$ margin was performed and a free anterolateral thigh (ALT) flap was used to reconstruct the defect. This technique satisfactorily reconstructed a large defect in an adolescent with minimal donor site morbidity, as the donor site was closed primarily, and a functionally and aesthetically acceptable outcome. There was no evidence of tumour recurrence at 6 months follow-up.

S Afr J Surg 2018;56(4)

http://dx.doi.org/10.17159/2078-5151/2018/v56n4a2849

\section{Case Report}

A 16-year-old male presented with a $5 \times 5 \mathrm{~cm}$ mass on the medial aspect of his right lower leg which had been present for 2 years (Figure A). Three core biopsies of the lesion demonstrated highly cellular tissue consisting of spindle shaped cells arranged in a storiform pattern, consistent with DFSP. No fibrosarcomatous change or malignant transformation was noted. Immunohistochemistry was diffusely positive for CD34 and positive for Factor VIIIa. An MRI of the lower leg revealed a well-circumscribed superficial anterior calf mass arising from the subcutaneous tissue with three ovoid intralesional nodules. No bony, muscular or neurovascular involvement was demonstrated.

Surgical treatment consisted of a wide local excision (WLE) with a $2 \mathrm{~cm}$ margin, incorporating the deep fascia and periosteum of the tibia (Figure B). A $9 \times 11 \mathrm{~cm}$ free anterolateral thigh flap was raised on a single musculocutaneous perforator supplied by the descending branch of the lateral circumflex artery of the thigh as pedicle (Figure C). The recipient vessels were the posterior tibial artery and venae commitantes. The flap was tailored to cover the defect, without any tension (Figure D). The donor site was closed primarily. Postoperative course was uneventful and the patient mobilised fully 5 days after surgery. The patient was discharged 10 days after surgery. Postoperative histology revealed clear surgical margins, although the spindle cells revealed mild nuclear atypia and were mitotically active. No postoperative radiotherapy was indicated following discussion at the multi-disciplinary meeting. There was no evidence of recurrence at 6 months follow-up.

\section{Discussion}

Dermatofibrosarcoma protuberans (DFSP) is a rare, slow growing soft tissue tumour which is locally aggressive with a low potential for metastatic spread. First described in 1890 by Taylor, DFSP was only recognised as a distinct cutaneous entity by Darier and Ferrand in 1924. At that stage it was known as a progressive and recurring dermatofibroma. Hoffman officially presented the term DFSP. ${ }^{1}$ The incidence of DFSP in the adolescent years (10-19 years of age) is rare $(1.3 \%)$. There is no gender preponderance and $18 \%$ of tumours occur on the lower extremity. ${ }^{2}$ The pathogenesis of DFSP is still unknown, although evidence suggests that pluripotent undifferentiated mesenchymal cells may lead to the development. ${ }^{3}$ Due to its slow growth and mostly asymptomatic progression, tumours can present at a considerable size as seen in our case report. Tumours are usually locally destructive, with high recurrence rates and low metastatic spread. ${ }^{2,4,5}$ Diagnosis is usually due to high clinical suspicion, followed by histologically proven incisional biopsy. Imaging is seldomly needed, but preoperative magnetic resonance imaging (MRI) is useful for the assessment of larger and more atypical tumours. ${ }^{6}$

Surgical excision with at least a $2-3 \mathrm{~cm}$ margin remains the gold standard of treatment, while radiotherapy is mostly used for adjuvant treatment. ${ }^{7}$ Mohs micrographic surgery and wide local excision have been shown to have similar recurrent rates, although Mohs micrographic surgery has fewer positive margins. ${ }^{7}$ Tsai et al. reported no recurrence rates in 13 adolescent patients with DFSP over a 20 month to 19-year period by using WLE with $3 \mathrm{~cm}$ margins. ${ }^{5}$ In cases of unresectable, recurrent and/or metastatic disease a protein tyrosine kinase inhibitor, namely Imatinib mesylate has been approved. $^{8}$ 


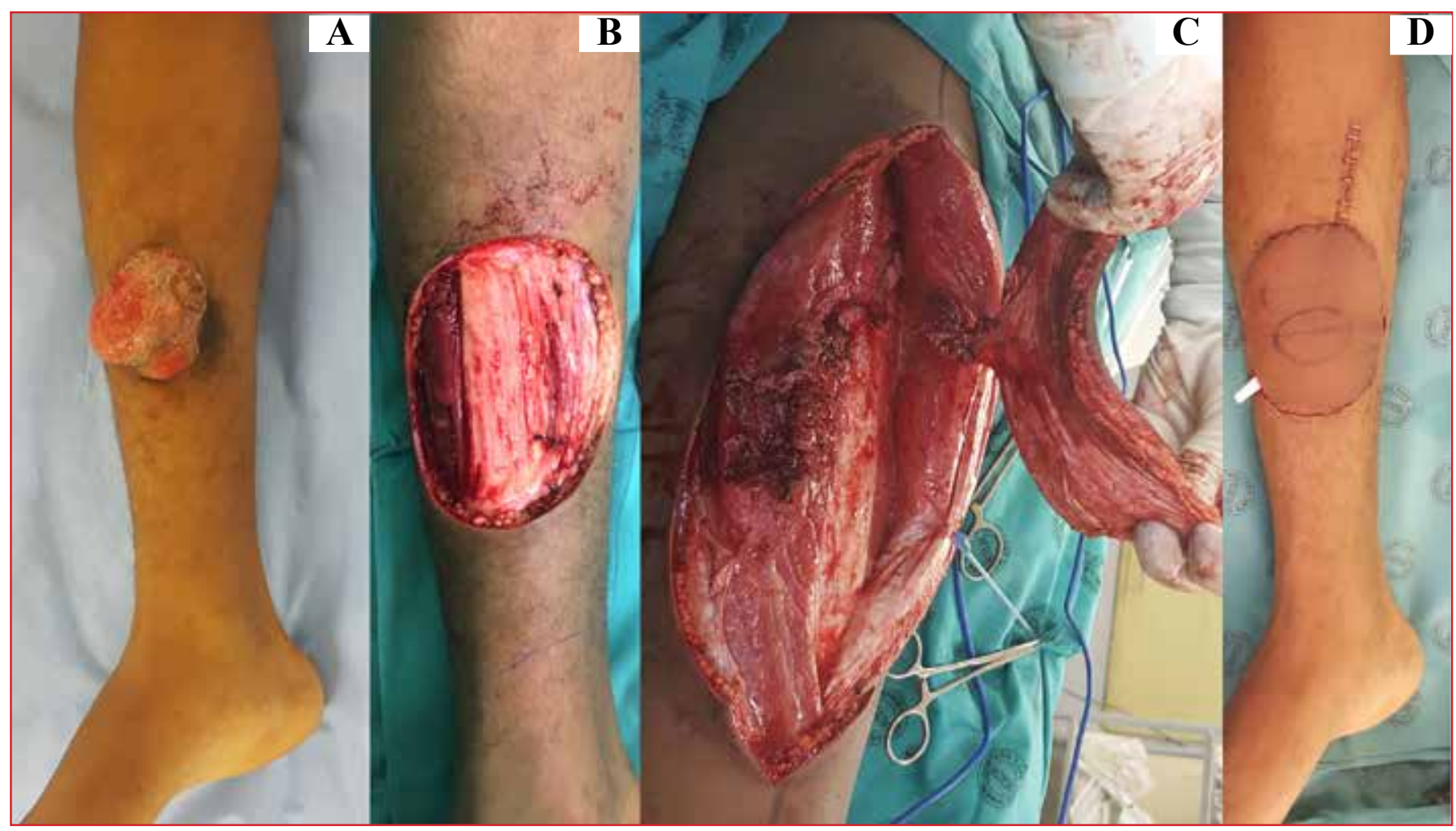

Figures: Composite Picture of DFSP excision and reconstruction

Reconstructing the defect after surgical excision can be challenging. Options range from simple split thickness skin graft (SSG) to free tissue transfer. Loco-regional and free flaps provide better results than SSG in terms of soft tissue cover, functional outcome and aesthetic results. ${ }^{9}$ Unfortunately, local flaps can lead to additional scars at the excision site and for this reason we prefer to reconstruct the defect utilising free tissue transfer.

As in our case report, the versatility and location of the anterolateral thigh flap makes it well suited for lower extremity reconstruction after both traumatic and oncological surgery. ${ }^{10}$ It provides robust cover, minimal donor site morbidity as most donor sites can be closed primarily with an aesthetically acceptable scar and better functional outcome than its locoregional counterparts.

\section{Conclusion}

Although exceedingly rare, DFSP can present as a locally destructive tumour in an adolescent. Early histological diagnosis by incisional biopsy is mandatory, as the reconstruction of the defect after wide local excision can be challenging. Loco-regional or free flaps provide better cover for the defect than split thickness skin grafts. A free anterolateral thigh flap can be successfully used to reconstruct larger defects with minimal patient morbidity and an acceptable aesthetic outcome.

\section{REFERENCES}

1. Hoffman E. Uber das knollentreibende fibrosarkom de haut. Dermatol Z. 1925;43:128.
2. Criscione VD, Weinstock MA. Descriptive epidemiology of dermatofibrosarcoma protuberans in the United States, 1973 to 2002. J Am Acad Dermatol. 2007;56(6):968-973. PMID: 17141362. doi: 10.1016/j.jaad.2006.09.006

3. Dimitropoulos VA. Dermatofibrosarcoma protuberans. Dermatol Ther. 2008;21(6):428-32. PMID: 19076619. doi: 10.1111/j.1529-8019.2008.00242.x

4. Trofymenko O, Bordeaux JS, Zeitouni NC. Survival in patients with primary dermatofibrosarcoma protuberans: National cancer database analysis. J Am Acad Dermatol. 23 Nov 2017;S0190-9622(17)32729-9. PMID: 29175214. doi: 10.1016/j.jaad.2017.11.030

5. Tsai Y, Lin P, Chew K, Chiang Y. Dermatofibrosarcoma protuberans in children and adolescents: Clinical presentation, histology, treatment, and review of literature. Journal of Plastic, Reconstructive and Aesthetic Surgery.2014;67:1222-9. PMID: 24973861. doi: 10.1016/j.bjps.2014.05.031

6. Thornton SL, Reid JE, Papay FA, Vidimos AT. Childhood dermatofibrosarcoma protuberans: role of preoperative imaging. Journal of the American Academy of Dermatology. 2005;53:76-83. PMID:15965425 doi:10.1016/j. jaad.2004.11.071

7. Meguerditchian AN, Wang J, Lema B, Kraybill WG, Zeitouni NC, Kane JM 3rd. Wide excision or Mohs micrographic surgery for the treatment of primary dermatofibrosarcoma protuberans. Am J Clin Oncol.2010;33(3):300-3. PMID: 19858696. doi: 10.1097/COC.0b013e3181aaca87

8. McArthur GA. Molecular targeting of dermatofibrosarcoma protuberans: a new approach to a surgical disease. J Natl Compr Canc Netw. 2007;5:557-62. PMID: 17509258

9. Chan T, Wu C, Jeng S. Dermatofibrosarcoma protuberans: A 10year experience. Formosan Journal of Surgery.2015;48:10-16. doi:http://dx.doi.org/10.1016/j.fjs.2014.06.004

10. Nosrati N, Chao AH, Chang DW, Yu P. Lower extremity reconstruction with the anterolateral thigh flap. J Reconstr Microsurg. May 2012;28(4):227-34. PMID: 22399252. doi: $10.1055 / \mathrm{s}-0032-1306370$. 